\title{
T-cell Lymphoblastic Leukaemia/Lymphoma Presenting as Bilateral Renal Enlargement and Arthritis: A Rare Case Report
}

\author{
Md. Minhaj Uddin Bhuiyan, ${ }^{1}$ Rajib Bhowmic, ${ }^{2}$ Md. Shahriar Siddiki, ${ }^{3}$ Md. Kamrul Hasan Patwari, ${ }^{3}$ Sarmistha \\ Biswas, ${ }^{4}$ Md. Faizul Islam Chowdhury ${ }^{5}$
}

\begin{abstract}
Lymphoma usually presents as painless enlargement of lymph nodes with or without systemic symptoms like fever, weight loss, night sweats, itching and hepatosplenomegaly. But renal enlargement and arthritis as initial manifestations of lymphoma are very uncommon and poses a potential diagnostic challenge. Renal manifestations of lymphoma are usually nonspecific hematuria, fever, flank pain and oliguria. Pathological data are scanty in this regard; few reports indicate that it has a very poor prognosis. Here we described a case of lymphoma presented with bilateral palpable kidneys, pyrexia and arthritis. Initially diagnosis was confused as renal dysfunction was absent and also the condition is rare. However, strong clinical suspicion along with radiological and histopathological evidence as well as immunophenotyping tests helped to diagnose the case as T-cell lymphoblastic leukaemiallymphoma.
\end{abstract}

Keywords: Lymphoma, leukaemia, palpable kidneys, arthritis, immunophenotyping

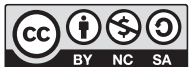

DOI: https://doi.org// 0.3329/jom.v2 Ii2.502 I6

Copyright: (c) 2020 Bhuiyan MMU et al. This is an open access article published under the Creative Commons Attribution-NonCommercial-NoDerivatives 4.0 International License, which permits use, distribution and reproduction in any medium, provided the original work is properly cited, is not changed in any way and it is not used for commercial purposes.

Received: 44 February 2019;

Accepted: 03 September 2020

\section{Introduction}

Acute lymphoblastic leukaemia (ALL)/lymphoblastic lymphoma (LBL) is a neoplasm of lymphoblasts committed to either T-cell or B-cell lineage and involves bone marrow and blood as defined by World Health Organization classification. ${ }^{1}$

Conventionally, the term lymphoma is used when the process is confined to a mass lesion with no/minimal evidence of peripheral blood and bone marrow involvement. The term leukemia is used when there is extensive peripheral blood and bone marrow involvement. LBL accounts for approximately $2 \%$ of all lymphomas. ${ }^{2}$ Both subtypes of ALL/ LBL are morphologically similar on light microscopy (LM) and require immunophenotyping for differentiation.

1. Registrar, Department of Medicine, Dhaka Medical College Hospital (DMCH), Dhaka

2. Assistant Registrar, Department of Medicine, DMCH, Dhaka

3. Indoor Medical Officer, Department of Medicine, DMCH, Dhaka

4. Associate Professor, Department of Medicine, DMCH, Dhaka

5. Professor, Department of Medicine, DMCH, Dhaka

Corresponding author: Dr. Md. Minhaj Uddin Bhuiyan, Registrar, Department of Medicine, Dhaka Medical College Hospital (DMCH), Dhaka. Email: mubrussell@gmail.com
LBL can be an extra-nodal disease, with a propensity to involve mediastinum, skin, bone, liver, spleen, testis, and central nervous system. ${ }^{1}$ Kidney as a sole site of involvement is extremely rare; fewer than 100 cases of lymphoma diagnosed by percutaneous kidney biopsy have been reported. Diffuse large B-cell lymphoma is the most common subtype reported and precursor LBL/ALL accounts for very few cases. ${ }^{2}$

\section{Case summary}

A 27-year-old male admitted in a tertiary care hospital with the complaints of arthralgia for 4 months and fever for 3 months. According to the statement of the patient, he was reasonably well 4 months back. One night, he suddenly developed severe right elbow pain which awakened him from sleep. The pain was not associated with any swelling or redness and subsided gradually after taking medications. Then about 2 weeks later, he again suddenly developed polyarthritis including metacarpophalangeal (MCP), interphalangeal (PIP) and wrist joints of both upper limbs and metatarsophalangeal (MTP), ankle and knee joints of both lower limbs with significant morning stiffness. Pain was associated with swelling and redness, aggravated by taking rest and relieved after activities and taking analgesics. $\mathrm{He}$ 
also complained of pain in shoulder and elbow joints without any associated swelling or redness. Patient also complained of recurrent bouts of fever for last 3 months. Each episode persisted for 5-6 days. Fever was continued in nature, not associated with chills, rigor or night sweats and subsided after taking medications. Highest recorded temperature was $103^{0} \mathrm{~F}$. Patient also complained of hair loss, recurrent painless oral ulcers and significant weight loss of about $10 \mathrm{~kg}$ within last 4 months. His bowel and bladder habit is normal. There is no history of rash, photosensitivity, convulsion, cough, abdominal pain or back pain. Patient took non-steroidal antiinflammatory drugs (NSAIDs), disease modifying antirheumatic drugs (DMARDs) and steroids. There is no such type of illness in his family.

On examination, patient was anaemic with diffuse nonscarring alopecia on scalp and cervical lymphadenopathy involving both posterior chain and post-auricular region with largest one measuring about $1.5 \mathrm{~cm} \mathrm{X} 1 \mathrm{~cm}$ smooth, mild tender, firm in consistency, mobile and there is no discharging sinus. Pulse: 90 beats per minute \& regular, BP: 130/80 mm $\mathrm{Hg}$ with no postural drop, Temperature: $103^{\circ} \mathrm{F}$, Respiratory rate: 20 breaths/ minute. Abdomen examination revealed just palpable liver and bilaterally palpable kidneys. Musculoskeletal system examination revealed swelling, redness, tenderness and restriction of both active and passive movements in MCP, wrist, PIP, MTP, ankle and knee joints.

Investigation showed anaemia and thrombocytopenia with leukocytosis. Urine R/M/E, S. electrolytes were normal. Serum Creatinine $1.8 \mathrm{mg} / \mathrm{dl}$. USG of whole abdomen and later CT abdomen with contrast [Figure 1] both revealed mild hepatomegaly with bilateral diffusely enlarged kidneys. Rheumatoid arthritis factor, Anti-CCP antibody, ANA, AntiHIV antibodies are negative. S. LDH $3138 \mathrm{U} / \mathrm{L}$, uric acid $12.80 \mathrm{mg} / \mathrm{dl}$. Lymph node excision biopsy showed loss of nodal architecture with diffuse infiltration of large round cells, mitoses were noted. Findings were consistent with diffuse large cell non-Hodgkin lymphoma, high grade. Bone marrow study showed some cellular elements, majority cells are blasts having scanty cytoplasm, high $\mathrm{N}: \mathrm{C}$ ratio, coarse chromatin \& inconspicuous nucleoli resembling lymphoblast. These features were suggestive of acute lymphoblastic leukaemia. Immunohistochemistry (lymph node) showed CD3, CD1a, CD7, CD4, Tdt (EP-266) diffusely positive and Ki-67 expression was 85-90\% [Figure 2]. Diagnosis was consistent with adult T-cell lymphoblastic leukaemia/ lymphoma. The patient was referred to the department of hematology for further management.

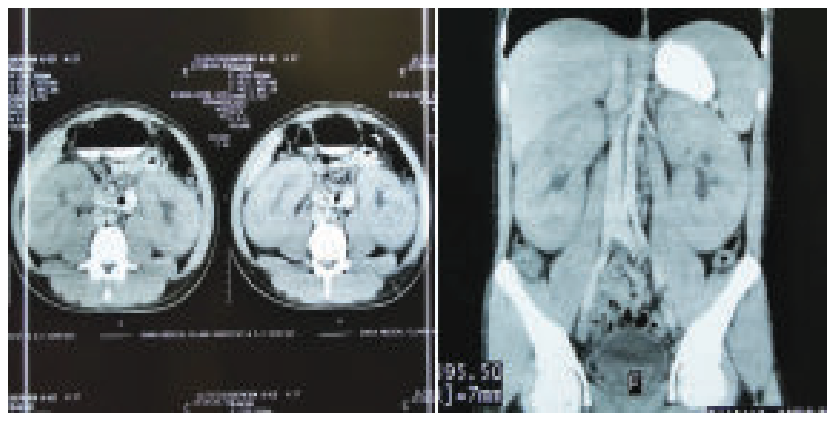

Fig.-1 : CT Abdomen shows diffuse enlargement of both kidneys.
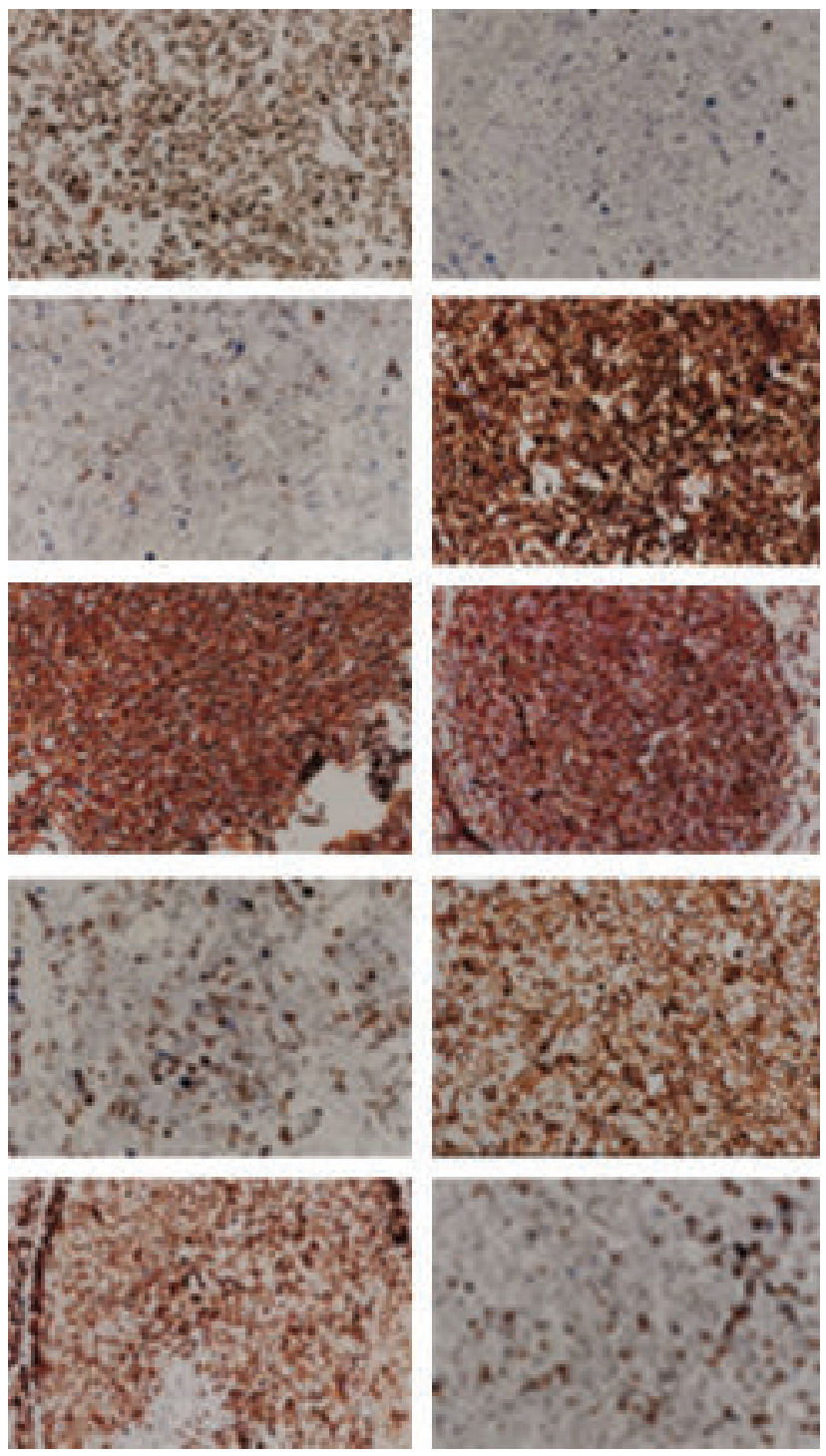

Fig.-2 : Immunohistochemistry shows CD3, CD1a, CD7, CD4, Tdt (EP-266) diffusely positive \& Ki-67 expression was $85-90 \%$. CD20, CD2, CD8 \& CD5 were negative, confirming the diagnosis T-cell lymphoblastic lymphomal leukaemia. 


\section{Discussion}

The first description of lymphoma diagnosed by percutaneous renal biopsy was published in 1980 by Coggins. ${ }^{3}$ There seems to be a variation in clinical presentation of the subtypes of lymphomas. B-cell LBL frequently involves the skin, bone, soft tissue, lymph nodes, ovaries, retroperitoneum, and tonsils. On the other hand, Tcell LBL usually presents as a mediastinal mass or with lymphadenopathy in cervical, supraclavicular, and axillary regions. Renal involvement usually occurs late in the course of the disease and is clinically silent. Very few cases have been reported in the literature of LBL diagnosed by kidney biopsy. ${ }^{2}$

B-LBL/ALL is morphologically indistinguishable from precursor T- LBL/ALL on light microscopy, but differentiation is based on the expression of lineage specific markers (immunophenotyping). ${ }^{4}$

The clinical suspicion of lymphomatous infiltration should arise when patients present with unexplained AKI and/or bilateral nephromegaly. While other causes of increased renal size can be considered, a definitive diagnosis requires a renal biopsy. The indications for biopsy in previous case reports were unexplained renal failure or proteinuria except for few cases where the indication was bilateral nephromegaly detected on radio imaging. ${ }^{3}$

Radiographic interpretation of renal lymphoma is difficult and needs skill and experience. Contrast enhanced $\mathrm{CT}$ is the preferred method for diagnosing renal lymphoma, but has the disadvantage of nephrotoxicity. Recently, magnetic resonance imaging has been proposed to be superior, especially in patients with renal failure, in diagnosis of lymphoma. ${ }^{5}$

There is considerable doubt among pathologists over development of lymphoma in kidneys, as kidneys do not have identifiable lymphatic channels. Some pathologists tend to think it is a disseminated malignancy, because in 10 to $20 \%$ of cases lymphoma affects both kidneys, as was in the present case. Mucosa-associated lymphoid tissue renal lymphoma is probably the underlying mechanism. ${ }^{6}$

In this case, diagnosis was made on the basis of histology and immunohistochemistry of lymph node and bone marrow study with flow cytometry. Renal biopsy was planned but couldn't be done due partly to patients general ill health and already established diagnosis by lymph node and bone marrow studies. However, fine needle aspiration or core renal biopsy clearly is the best method to establish diagnosis with high specificity and sensitivity. ${ }^{6}$
Leukaemia, and more rarely other tumours such as lymphoma, may present with musculoskeletal manifestations, however, true articular signs such as joint swelling are less frequent, and have been reported only rarely at the onset of lymphoma. ${ }^{7,8}$

The presence of joint swelling in leukaemia is more frequent in children than in adults, and in acute rather than in chronic forms. Proposed pathogenetic mechanisms include: infiltration of leukaemic cells into bone or synovial tissue, haemorrhage into the joints secondary to thrombocytopenia, joint infection, gouty arthritis, synovial reaction to periosteal or capsular infiltration and immune complex induced synovitis. ${ }^{9}$ In our patient, serum uric acid was found elevated.Synovial immunocytology was not performed in our patients; however few studies on synovial fluids in leukaemia/lymphoma and arthritis have shown variable leucocyte counts and cytologic characteristics. ${ }^{10}$

Treatment of acute T-cell lymphoblastic lymphoma/leukemia with renal involvement remains controversial because pathogenetic mechanisms are uncertain. Role of radiation as a therapy in treatment of bilateral diffuse lymphoma infiltration is not established. It is equally unclear if it should be only treated by chemotherapy or in combination with radiation. Lack of therapeutic trials in literature has obviously compelled clinicians to adopt a practical approach and treat according to histological grade. As it has been established that most cases are high-grade lymphoma, they are treated by CHOP or other chemotherapy regimen with or without radiotherapy. ${ }^{11,12}$ Rituximab was not used as it is not B-cell type. However, results are usually unsatisfactory due to rapid progression of disease and treatment resistance. ${ }^{6}$

\section{Conclussion}

Herein, we reported a case of lymphoma presenting with bilateral enlarged kidneys and polyarthritis, which poses significant confusion to reach a diagnosis due to the atypical presentation. Physicians should consider lymphoma as cause of renomegaly if clinical suspicion is present and other differentials are excluded.

\section{Conflict of interest: None.}

\section{References}

1. Borowitz MJ, Chan JK. T lymphoblastic leukemia/ lymphoma. In: Swerdlow S, Campo E, Lee Harris N, Jaffe ES, Pileri SA, Stein H, et al., editors. WHO Classification of Tumours of Haematopoietic and Lymphoid Tissues. Lyon: IARC; 2008. p. 168-78

2. Törnroth T, Heiro M, Marcussen N, Franssila K. Lymphomas diagnosed by percutaneous kidney biopsy. Am J Kidney Dis 2003;42:960-71 
3. Coggins $\mathrm{CH}$. Renal failure in lymphoma. Kidney Int 1980; $17: 847-55$

4. Boucheix C, David B, Sebban C, Racadot E, Bené MC, Bernard A, et al. Immunophenotype of adult acute lymphoblastic leukemia, clinical parameters, and outcome: An analysis of a prospective trial including 562 tested patients (LALA87). French group on therapy for adult acute Lymphoblastic Leukemia. Blood 1994;84:1603-12

5. Sheeran SR, Sussman SK. Renal lymphoma: Spectrum of CT findings and potential mimics. AJR Am J Roentgenol 1998; $171: 1067-72$

6. Tuzel E, Mungan MU, Yorukoglu K, Basakci A, Kirkali Z. Primary renal lymphoma of mucosa associated lymphoid tissue. Urology. 2003;61:463-4

7. Ueno Y, Manabe T, Shimizu S. Non-Hodgkin's lymphoma and polyarthritis. Br J Rheumatol 1995;34:293-7.

8. Evans T1, Nercessian BM, Sanders KM. Leukemic arthritis. Semin Arthritis Rheum 1994;24:48-56.

6. Howard D. Dorfman, Howard L. Siegel, Michael C. Perry, Oxenhandler R. Non-hodgkin's lymphoma of the synovium simulating rheumatoid arthritis. Arthritis Rheum 1987; Vol: 30 No. 2: $155-160$

7. Birlik M, Akar S, Onen F, Ozcan A. Mehmet, Bacakoglu A, Ozkal S, et al. Articular, B-cell, non-Hodgkin's lymphoma mimicking rheumatoid arthritis: synovial involvement in a small hand joint. Rheumatol Int 2004; 24: 169-172

8. Mcdonagh JE, Clarke F, Smith SR, Kesteven P, Walker J. Non-hodgkin's lymphoma presenting as polyarthritis. Rheumatology (Oxford) 1994;33:79-84

9. Martin VM, Bennett RM, Hughes GR, Holt PJ. Lymphosarcomatous arthropathy .Ann Rheum Dis. 1973; 32: $162-166$

10. Fam AG, Voorneveld C, Robinson JB, Sheridan BL. Synovial fluid immunocytology in the diagnosis of leukemic synovitis. J Rheumatol 1991;18:292-6

11. Saito S. Primary renal lymphoma: Case report and review of the literature. Uro Int. 1996;56:192-5

12. Stallone G, Infante B, Manno C, Campobasso N, Pannarale G, Schena FP. Primary renal lymphoma does exist. J Nephrol. 2000;13:367-72. 